\title{
PREDICTION OF LIVE BODY WEIGHT THROUGH BIRD AGE AND/OR SHANK LENGTH IN DIFFERENT FOWL TYPES
}

\author{
Ensaf A. El Full
}

Poult. Prod. Dept., Faculty of Agriculture, Cairo University, Fayoum Branch.

\begin{abstract}
:
This study was designed to describe and to fit linear statistical models to predict live body weight (LBW) through shank length (SL) and LBW or SL through bird age in three breeds of chickens: Fayoumi (PP), Golden Montazah (GM) and Dandarawi (Dand) and two breeds of ducks: Pekin and Moscuvy and Japanese quail. The data of SL in 'mm' and LBW in grams were collected from each bird in biweekly intervals up to 12 weeks of age for chickens, ten weeks for ducks and up to 6 weeks of age for quail.

The following results were obtained

1. There was a definite relationship $(\mathrm{P} \leq 0.001)$ between LBW and SL for all studied sex groups, except for Moscuvy males. SL was positively correlated with LBW ranging from 0.794 to 0.977 .

2. As SL increased by one $\mathrm{mm}$ for studied groups, LBW progressively $(\mathrm{P} \leq 0.001$ ) increased ranging from $9.12 \mathrm{~g}$ (Dandarawi males) to 55.41g (Pekin females), indicating that $63 \%$ to $95.5 \%$ of the variation in LBW were explained by SL.

3. The cubic form had higher value for coefficient of determination ' $\mathrm{R}$ ', and lower standard error of estimate 'SE' than quadratic, logarithmic and exponential models in Moscuvy males. The cubic and quadratic forms had the best fit for predicting LBW from SL for ducks males, regardless of ducks breed.

4. Age in days significantly increased $(\mathrm{P} \leq 0.001) \mathrm{LBW}$ of different types of fowl of both sexes or each sex separately. All groups showed positive regression coefficient ranging from $4.320 \mathrm{~g} / \mathrm{one}$-day in case of Japanese quail males to $59.499 \mathrm{~g} /$ one-day in case of Moscuvy males.

5. Regardless of breed, males of both chickens and ducks had higher regression coefficients than their females. On the contrary, females in the case of Japanese quail showed higher rate of increase of LBW as influenced by age than their males.

6. Similarly, trends of positive significant influence of age in days on SL in millimeters but lower in magnitude than LBW were observed in most studied groups or sex groups.

7. There were definite relationships between LBW and SL and bird age significantly affected either LBW or SL. Comparing ' $\mathrm{R}$ ', of fitted equations indicated that linear model was found to be suitable for predicting LBW through bird age (with $\mathrm{R}^{2}$ ranging from 0.809 to 0.966 ) or SL (with $\mathrm{R}^{2}$ ranging from 0.630 to 0.955 ).

8. In the absence of age, shank length can be used to predict live body weight.
\end{abstract}

Key Words: Prediction -Live body weight - Age - Shank length - Fowl.

Fayoum J. Agric. Res. \& Dev., Vol.19, No.1, January, 2005 


\section{INTRODUCTION}

The development of a broiler industry requires knowledge of the genetic aspects of growth pattern for different breeds and lines and their hybrids so that they might be selected for specific types of production (Knizetova et al., 1994). Breed differences in six breeds of chickens were detected by Jaap and Thomson (1940) for the ratio between shank length (SL) and live body weight (LBW). Wise (1970) noted that broilers had similar proportions by weight of the various body parts as layers when compared at the same weight, however, broilers had shorter bones and more compact body parts. But not much work had been undertaken to predict LBW from SL in other types of poultry.

The use of live body measures as indicators of conformation were advocated by Jaap and Penquite (1938). Various investigations conducted to find out the correlation between LBW and SL in poultry. Kanoun (1984) tried to predict LBW of chicken through body measurements. Verma et al. (1977) and Tierce and Nordskog (1985) and Sunanda et al. (1991) used different statistical models to predict LBW from SL of Desi ducks and regression equations were fitted for predicting LBW from SL in ducks. Similarly, Shanawany and Morris (1992) reported that body weight linearly related to shank length of chickens. Many of the body measurements including SL are good indicators of skeletal size (Chambers, 1993 and El Full and Farahat, 2004). Several investigators reported that SL had generally high positive genetic and phenotypic correlations with various economic traits (Petek et al., 2000,Singh $\boldsymbol{e t}$ al., 2001 and Kumar $\boldsymbol{e t}$ al., 2002). Therefore, shank length affected the layer performance and would be a very useful method for estimating pullet value.

Models, which are nonlinear in the parameters, are intrinsically linear if a transformation will make them linear, logarithmic and exponential curves are typical examples as illustrated by Steel and Torrie (1981).

This study was initiated to describe and to fit linear statistical models to predict LBW through SL in six genetic groups of Japanese quail, ducks and Egyptian chickens and to predict LBW or SL through bird age.

\section{MATERIALS AND METHODS}

This work was carried out in the Poultry Research Station, Poultry Production Department, Faculty of Agriculture at Fayoum, Cairo University during the period from August 2002 to November 2003. All chicks were wingbanded, immunized for Marek's disease immediately after hatching and brooded in floor brooders. The appropriate feeding, vaccination and management practices were kept uniform as possible throughout the experimental period for each type of fowl throughout the experimental period. Chickens fed the same recommended standard diets that appropriate for each type and age. Feed and water were offered ad lib. The minerals and vitamins were adequately supplied to cover the requirements according to the Egyptian Ministerial Decree No. 1498 (1996) and NRC (1994). 
PREDICTION OF LIVE BODY WEIGHT THROUGH BIRD AGE

This study was initiated using the following numbers of different genetic stocks:

\begin{tabular}{|l|c|c|c|}
\hline Fowl type & Males & Females & Males + Females \\
\hline Chickens: & & & \\
Fayoumi ,PP & 999 & 1070 & 2069 \\
Golden Montazah, GM & 844 & 905 & 1749 \\
Dandarawi, Dand & 1323 & 1510 & 2833 \\
All chickens & 3166 & 3485 & 6651 \\
\hline Pekin ducklings & 62 & 65 & 127 \\
Moscuvy ducklings & 72 & 88 & 160 \\
All ducks & 134 & 153 & 287 \\
\hline Japanese quail & 133 & 141 & 274 \\
\hline Total fowl types & 3433 & 3779 & 7212 \\
\hline
\end{tabular}

PP: A line of Fayoumi selected for high egg production in the first 90 days of production .

The data of SL in ' $m m$ ' and LBW in grams were collected from each bird in biweekly intervals up to 12 weeks of age for chickens, ten weeks for ducks and up to 6 weeks of age for quail.

This study was initiated using shank growth of different types of fowl for predicting live LBW traits. Separate growth models were presented for both sexes together and for each sex separately. The following regression models were used:

\begin{tabular}{|c|c|c|}
\hline No. & Model & Description \\
\hline 1 & \multicolumn{2}{|c|}{ Polynomial linear models } \\
\hline 1.1. & Linear 1 & $\begin{array}{l}\mathrm{Y}=\mathrm{b} 0+\left(\mathrm{b} 1^{*} \mathrm{X}\right) \text {. } \\
\text { Where ' } \mathrm{Y} \text { ': is the predicted LBW, ' } \mathrm{X} \text { ': is the SL, 'b0' and ' } \mathrm{b} \text { ' are the } \\
\text { constants to be determined through method of least squares. } \\
\mathrm{Y}=\mathrm{b} 0+\mathrm{b} 1^{*}(\mathrm{X}) \text {. }\end{array}$ \\
\hline 1.2 . & Linear 2 & $\begin{array}{l}\text { Where ' } \mathrm{Y} \text { ': is the predicted LBW, ' } \mathrm{X} \text { ': is the bird age, 'b0' and ' } \mathrm{b} \text { ' } \\
\text { are the constants to be determined through method of least squares. } \\
\mathrm{Y}=\mathrm{b} 0+\mathrm{b}^{*}(\mathrm{X}) \text {. } \\
\text { Where ' } \mathrm{Y} \text { ': is the predicted SL, ' } \mathrm{X} \text { ': is the bird age, ' } \mathrm{b} 0 \text { ' and ' } \mathrm{b} \text { ' are } \\
\text { the constants to be determined through method of least squares. }\end{array}$ \\
\hline 2 & Quadratic & $\begin{array}{l}\mathrm{Y}=\mathrm{b} 0+(\mathrm{b} 1 \mathrm{X})+\left(\mathrm{b} 2 * \mathrm{X}^{* * 2}\right) \\
\text { This model can be used to model a series which "takes off" or a } \\
\text { series which dampens. }\end{array}$ \\
\hline 3. & Cubic & $\mathrm{Y}=\mathrm{b} 0+\left(\mathrm{b} 1{ }^{*} \mathrm{X}\right)+\left(\mathrm{b} 2 * \mathrm{X}^{* 2}\right)+\left(\mathrm{b} 3 * \mathrm{X}^{* * 3}\right)$ \\
\hline 4. & \multicolumn{2}{|c|}{ Nonlinear models } \\
\hline 4.1. & Logarithmic & $\mathrm{Y}=\mathrm{b} 0+\left(\mathrm{b} 1{ }^{*} \ln (\mathrm{X})\right)$ \\
\hline 4.2. & Exponential & $\mathrm{Y}=\mathrm{b} 0^{*}\left(\mathrm{e}^{* *\left(\mathrm{bl}{ }^{*} \mathrm{X}\right)}\right)$ or $\ln (\mathrm{Y})=\ln (\mathrm{b} 0)+\left(\mathrm{b} 1^{*} \mathrm{X}\right)$. \\
\hline
\end{tabular}

In order to compare the relative efficiency of various growth curve models and to select the most suitable curve, the following two criteria were used. One is the coefficient of determination $\left(\mathrm{R}^{2}\right)$ and the other is standard error (SE). A larger value of $R^{2}$ and smaller value on $S E$ indicate best fit of the curve.

\section{RESULTS AND DISCUSSION}

Analyses of variance of both LBW and SL between males and females were applied for each age within each studied group or type. The results showed a significant sex effect favoring males than females for either LBW or SL at all ages studied in PP, Dand, GM and Moscuvy ducks (except for SL at 14 days of age). However, no significant differences were found between LBW of males and

Fayoum J. Agric. Res. \& Dev., Vol.19, No.1, January, 2005 
females for Pekin ducks at all ages and Japanese quail at 14 and 28 days of age. Females of Japanese quail showed significantly $(\mathrm{P} \leq 0.01)$ heavier LBW at 42 days of age than males.

As shown in Figure 1, males had heavier LBW $(\mathrm{P} \leq 0.01)$ for all chickens at all ages studied and all ducks, except for 14 days of age than females regardless of breed. Whereas, except for LBW at 42 days of age, Japanese quail showed no significant differences between LBW or SL of males and females as shown in Table 1.

Table 1. Means of live body weight (g) and shank length ( $\mathrm{mm})$ for different types of fowl at different ages.

\begin{tabular}{|c|c|c|c|c|c|c|c|}
\hline \multirow{2}{*}{$\begin{array}{r}\text { Group } \\
\text { Chickens: }\end{array}$} & \multirow{2}{*}{$\begin{array}{l}\text { Age, } \\
\text { days }\end{array}$} & \multicolumn{2}{|c|}{ Males } & \multicolumn{2}{|c|}{ Females } & \multicolumn{2}{|c|}{ Males + Females } \\
\hline & & LBW & SL & LBW & SL & LBW & SL \\
\hline \multirow[t]{3}{*}{ Fay, PP } & 28 & $215.69 \mathrm{~A}$ & $53.84 \mathrm{a}$ & 196.25B & $51.18 \mathrm{~b}$ & 205.97 & 52.51 \\
\hline & 56 & $485.56 \mathrm{~A}$ & $75.95 \mathrm{a}$ & $418.89 \mathrm{~B}$ & $70.64 \mathrm{~b}$ & 452.23 & 73.29 \\
\hline & 84 & $803.66 \mathrm{~A}$ & $93.76 \mathrm{a}$ & $650.63 \mathrm{~B}$ & $84.41 \mathrm{~b}$ & 727.14 & 89.08 \\
\hline \multirow[t]{3}{*}{ Dand } & 28 & $181.49 \mathrm{~A}$ & $50.89 \mathrm{a}$ & $161.61 \mathrm{~B}$ & $47.89 \mathrm{~b}$ & 171.55 & 49.39 \\
\hline & 56 & $504.14 \mathrm{~A}$ & $78.51 \mathrm{a}$ & $420.02 \mathrm{~B}$ & $71.98 \mathrm{~b}$ & 462.08 & 75.24 \\
\hline & 84 & $813.97 \mathrm{~A}$ & $96.01 \mathrm{a}$ & $653.19 \mathrm{~B}$ & $85.79 \mathrm{~b}$ & 733.58 & 90.90 \\
\hline \multirow[t]{3}{*}{ G M } & 28 & $278.68 \mathrm{~A}$ & $57.86 \mathrm{a}$ & $251.90 \mathrm{~B}$ & $54.91 \mathrm{~b}$ & 265.09 & 56.38 \\
\hline & 56 & $802.21 \mathrm{~A}$ & $90.88 \mathrm{a}$ & $660.39 \mathrm{~B}$ & $84.23 \mathrm{~b}$ & 731.30 & 87.56 \\
\hline & 84 & $1279.70 \mathrm{~A}$ & $110.29 a$ & $1032.69 \mathrm{~B}$ & $99.38 \mathrm{~b}$ & 1156.19 & 104.84 \\
\hline \multicolumn{8}{|l|}{ Ducks: } \\
\hline \multirow[t]{5}{*}{ Pekin } & 14 & $240.64 \mathrm{~A}$ & $47.50 \mathrm{a}$ & $271.63 \mathrm{~A}$ & $49.80 \mathrm{a}$ & 258.58 & 48.80 \\
\hline & 28 & $1060.88 \mathrm{~A}$ & $73.30 \mathrm{a}$ & $1078.45 \mathrm{~A}$ & $70.70 \mathrm{a}$ & 1071.05 & 71.80 \\
\hline & 42 & $1622.88 \mathrm{~A}$ & $80.00 \mathrm{a}$ & $1559.91 \mathrm{~A}$ & $72.40 \mathrm{~b}$ & 1586.42 & 75.60 \\
\hline & 56 & $2156.88 \mathrm{~A}$ & $80.80 \mathrm{a}$ & $2010.91 \mathrm{~A}$ & $75.30 \mathrm{~b}$ & 2072.37 & 77.60 \\
\hline & 70 & $2295.37 \mathrm{~A}$ & $89.10 \mathrm{a}$ & $2093.73 \mathrm{~A}$ & $79.30 \mathrm{~b}$ & 2178.63 & 84.20 \\
\hline \multirow[t]{5}{*}{ Moscuvy } & 14 & $278.87 \mathrm{~A}$ & $44.60 \mathrm{a}$ & $243.93 \mathrm{~B}$ & $43.50 \mathrm{a}$ & 253.75 & 43.90 \\
\hline & 28 & $828.77 \mathrm{~A}$ & $72.10 \mathrm{a}$ & $685.29 B$ & $62.20 \mathrm{~b}$ & 725.60 & 67.80 \\
\hline & 42 & $1402.65 \mathrm{~A}$ & $81.00 \mathrm{a}$ & 1315.82B & $76.60 \mathrm{~b}$ & 1340.22 & 78.80 \\
\hline & 56 & $2470.88 \mathrm{~A}$ & $96.30 \mathrm{a}$ & 2094.08B & $80.90 \mathrm{~b}$ & 2199.96 & 88.10 \\
\hline & 70 & $3590.32 \mathrm{~A}$ & $100.80 \mathrm{a}$ & $2827.38 \mathrm{~B}$ & $93.70 \mathrm{~b}$ & 3041.76 & 95.70 \\
\hline \multicolumn{8}{|l|}{ Japanese Quail } \\
\hline & 14 & $43.58 \mathrm{~A}$ & $27.60 \mathrm{a}$ & $47.57 \mathrm{~A}$ & $28.71 \mathrm{a}$ & 45.57 & 28.15 \\
\hline & 28 & $112.57 \mathrm{~A}$ & $38.73 \mathrm{a}$ & $119.27 \mathrm{~A}$ & $38.71 \mathrm{a}$ & 115.92 & 38.72 \\
\hline & 42 & $175.72 \mathrm{~B}$ & $40.53 \mathrm{a}$ & $189.86 \mathrm{~A}$ & $40.41 \mathrm{a}$ & 182.79 & 40.47 \\
\hline
\end{tabular}

A and B: mean values of LBW in the same age within the same group followed by different superscripts are significantly different at $\mathrm{P} \leq 0.01$, a and $\mathrm{b}$ : mean values of SL in the same age within the same group followed by different superscripts are significantly different at $\mathrm{P} \leq 0.01$.

The linear equation of the form $\hat{Y}=a+b X$ was fitted to predict the average LBW (grams) from the average SL $(\mathrm{mm})$ of different types of fowl. The values of the parameter ' $a$ ' and ' $b$ ', ' $r$ ', ' $\mathrm{R}^{2}$ ' and fitted equations were given in Table 2. 
PREDICTION OF LIVE BODY WEIGHT THROUGH BIRD AGE....... 45

Fig.

Fayoum J. Agric. Res. \& Dev., Vol.19, No.1, January, 2005 
Table 2. The parameters, coefficient of determination $\left(R^{2}\right)$ of $L B W$ when the linear equation $\hat{\mathrm{Y}}=\mathbf{a}+\mathbf{b} \mathrm{X}$ was used for predicting the average body weight in grams $(\hat{\mathbf{Y}})$ from average $S L$ in $\mathbf{m m}(X)$ of different types.

\begin{tabular}{|c|c|c|c|c|c|c|c|}
\hline Group & Sex & $\mathbf{a}$ & b & $\mathbf{r}$ & $\mathbf{R}^{2}$ & Sig. & Fitted equation \\
\hline \multicolumn{8}{|l|}{ Chickens: } \\
\hline \multirow[t]{3}{*}{ Fay, PP } & $\mathrm{M}$ & -575.579 & 14.421 & 0.966 & 0.933 & $* * *$ & $\hat{\mathrm{Y}}=-575.579+14.421 \mathrm{X}$ \\
\hline & $\mathrm{F}$ & -498.608 & 13.333 & 0.961 & 0.924 & $* * *$ & $\hat{\mathrm{Y}}=-498.608+13.333 \mathrm{X}$ \\
\hline & $\mathrm{M}+\mathrm{F}$ & -540.159 & 13.947 & 0.965 & 0.930 & $* * *$ & $\hat{\mathrm{Y}}=-540.159+13.947 \mathrm{X}$ \\
\hline \multirow[t]{3}{*}{ Dand } & $\mathrm{M}$ & -185.538 & 9.122 & 0.794 & 0.630 & $* * *$ & $\hat{\mathrm{Y}}=-185.538+9.122 \mathrm{X}$ \\
\hline & $\mathrm{F}$ & -446.352 & 12.515 & 0.962 & 0.926 & $* * *$ & $\hat{\mathrm{Y}}=-446.352+12.515 \mathrm{X}$ \\
\hline & $\mathrm{M}+\mathrm{F}$ & -292.595 & 10.407 & 0.861 & 0.741 & $* * *$ & $\hat{\mathrm{Y}}=-292.595+10.407 \mathrm{X}$ \\
\hline \multirow[t]{3}{*}{$\mathrm{G} \mathrm{M}$} & $\mathrm{M}$ & -814.493 & 18.546 & 0.977 & 0.955 & $* * *$ & $\hat{\mathrm{Y}}=-814.493+18.546 \mathrm{X}$ \\
\hline & $\mathrm{F}$ & -690.090 & 16.833 & 0.970 & 0.941 & $* * *$ & $\hat{\mathrm{Y}}=-690.090+16.833 \mathrm{X}$ \\
\hline & $\mathrm{M}+\mathrm{F}$ & -767.883 & 17.916 & 0.974 & 0.949 & $* * *$ & $\hat{\mathrm{Y}}=-767.883+17.916 \mathrm{X}$ \\
\hline \multirow[t]{3}{*}{ All chickens } & $\mathrm{M}$ & -485.962 & 13.662 & 0.938 & 0.880 & $* * *$ & $\hat{\mathrm{Y}}=-485.962+13.662 \mathrm{X}$ \\
\hline & $\mathrm{F}$ & -576.065 & 14.726 & 0.956 & 0.914 & $* * *$ & $\hat{\mathrm{Y}}=-576.065+14.726 \mathrm{X}$ \\
\hline & $\mathrm{M}+\mathrm{F}$ & -528.183 & 14.129 & 0.912 & 0.832 & $* * *$ & $\hat{\mathrm{Y}}=-528.183+14.129 \mathrm{X}$ \\
\hline \multicolumn{8}{|l|}{ Ducks: } \\
\hline \multirow[t]{3}{*}{ Pekin } & M & -2198.427 & 51.447 & 0.850 & 0.722 & $* * *$ & $\hat{\mathrm{Y}}=-2198.427+51.447 \mathrm{X}$ \\
\hline & $\mathrm{F}$ & -2442.928 & 55.410 & 0.845 & 0.714 & $* * *$ & $\hat{\mathrm{Y}}=-2442.928+55.410 \mathrm{X}$ \\
\hline & $\mathrm{M}+\mathrm{F}$ & -2286.749 & 52.898 & 0.848 & 0.718 & $* * *$ & $\hat{\mathrm{Y}}=-2286.749+52.898 \mathrm{X}$ \\
\hline \multirow[t]{3}{*}{ Moscuvy } & $\mathrm{M}$ & 1805.361 & 0.125 & 0.040 & 0.002 & NS & \\
\hline & $\mathrm{F}$ & -1531.427 & 38.893 & 0.897 & 0.805 & $* * *$ & $\hat{\mathrm{Y}}=-1531.427+38.893 \mathrm{X}$ \\
\hline & $\mathrm{M}+\mathrm{F}$ & 1468.992 & 0.273 & 0.071 & 0.005 & NS & \\
\hline \multirow[t]{3}{*}{ All ducks } & $\mathrm{M}$ & 1676.031 & 0.178 & 0.051 & 0.003 & NS & \\
\hline & $\mathrm{F}$ & -1609.286 & 40.967 & 0.863 & 0.744 & $* * *$ & $\hat{\mathrm{Y}}=-1609.286+40.967 \mathrm{X}$ \\
\hline & $\mathrm{M}+\mathrm{F}$ & 1446.367 & 3.831 & 0.079 & 0.006 & $* *$ & $\hat{\mathrm{Y}}=1446.367+3.831 \mathrm{X}$ \\
\hline \multirow[t]{3}{*}{ Japanese Quail } & $\mathrm{M}$ & -178.658 & 8.121 & 0.884 & 0.872 & $* * *$ & $\hat{\mathrm{Y}}=-178.658+8.121 \mathrm{X}$ \\
\hline & $\mathrm{F}$ & -231.154 & 9.740 & 0.869 & 0.755 & $* * *$ & $\hat{\mathrm{Y}}=-231.154+9.740 \mathrm{X}$ \\
\hline & $\mathrm{M}+\mathrm{F}$ & -203.512 & 8.900 & 0.871 & 0.759 & $* * *$ & $\hat{\mathrm{Y}}=-203.512+8.900 \mathrm{X}$ \\
\hline
\end{tabular}

Sig: significance, $* *$ : significantly different at $\mathrm{P} \leq 0.01,{ }^{* * *}$ : significantly different at $\mathrm{P} \leq 0.001$, NS: not significant.

The significance of regression and correlation coefficients showed that there were definite relationships $(\mathrm{P} \leq 0.001)$ between LBW and SL for all studied sex groups of chicken breeds, Pekin ducks and Moscuvy females, except for Moscuvy males (Table 2). SL was positively correlated with LBW ranging from 0.794 to 0.977 as shown in Table 2. Similar trends of using SL measurements for predicting LBW were reported in chickens (Kanoun, 1984), Desi ducks (Verma et al., 1977, Tierce and Nordskog, 1985 and Sunanda et al., 1991). Moscuvy males had lower insignificant $(\mathrm{P}>0.05)$ coefficient of determination than females of the two groups. However, as SL increased by one $\mathrm{mm}$ for other studied groups (chickens, ducks and Japanese quail), LBW progressively $(\mathrm{P} \leq 0.001)$ increased ranging from 9.122g (Dand' males) to $55.410 \mathrm{~g}$ (Pekin females). The corresponding $\mathrm{R}^{2}$ which ranged from 0.630 to 0.955 . In other words, SL positively increased LBW of these groups indicating that SL explained $63 \%$ to $95.5 \%$ of the variation in LBW.

Fayoum J. Agric. Res. \& Dev., Vol.19, No.1, January, 2005 
The values of the parameters ' $a$ ', ' $b$ ', coefficient of determination ' $\mathrm{R}^{2}$, standard error of estimate 'SE' and fitted equations when polynomial linear form (quadratic and cubic forms), logarithmic and exponential forms were used for predicting LBW from SL were given in Table 3. Moscuvy males had higher $\mathrm{R}^{2}$ and lower SE for polynomial models than logarithmic model. Similarly, the cubic form was better than the quadratic, logarithmic and exponential forms for predicting LBW from SL for ducks males, regardless of ducks breed. Because the value of $\mathrm{R}^{2}$, when cubic was used, was high and standard error of estimate was low as shown in Table 3.

It can be concluded that the cubic form was the best fit for predicting LBW from SL for either Moscuvy males and ducks males, regardless of ducks breed.

Age in days significantly increased $(\mathrm{P} \leq 0.001) \mathrm{LBW}$ of different types of fowl of both sexes or each sex separately. All groups showed positive regression coefficient which ranged from $4.320 \mathrm{~g} /$ one-day in case of Japanese quail males to $59.499 \mathrm{~g} /$ one-day in case of Moscuvy males. It can be seen that GM 'males, females and both sexes showed higher 'b's' (15.405, 12.309 and 13.911g) than either those of PP or Dand. Regardless of breed, males of both chickens and ducks had higher b's than their females. On the contrary, females in the case of Japanese quail showed higher rate of LBW increase as influenced by age than their males as shown in Table 4.

Table 3. The parameters, coefficient of determination $\left(R^{2}\right)$ and standard error of estimate (SE) of LBW when the polynomial or nonlinear equations were used for predicting the average body weight in grams $(\hat{Y})$ from average $S L(X)$ in $\mathbf{m m}$ for males of either Moscuvy or all ducks.

\begin{tabular}{|c|c|c|c|c|c|c|}
\hline $\begin{array}{c}\text { Curve } \\
\text { type }\end{array}$ & $\mathbf{a}$ & $\mathbf{B}$ & $\mathbf{R}^{2}$ & SE & Sig. & Fitted equation \\
\hline \multicolumn{7}{|c|}{ Moscuvy duck Males } \\
\hline \multicolumn{7}{|c|}{ Polynomial models: } \\
\hline Quadratic & -2366.172 & $\begin{array}{c}529.089 \\
-0.745\end{array}$ & 0.746 & 619.113 & $\begin{array}{l}* * * \\
* * *\end{array}$ & $\hat{\mathrm{Y}}=-2366.172+529.089 \mathrm{SL}-0.745 \mathrm{SL}^{2}$ \\
\hline Cubic & -2332.548 & $\begin{array}{c}518.619 \\
-0.001\end{array}$ & 0.747 & 617.883 & $\begin{array}{l}* * * \\
* * *\end{array}$ & $\begin{array}{l}\hat{\mathrm{Y}}=-2332.548+518.619 \mathrm{SL}-0.001 \mathrm{SL}^{3} \\
\left(\mathrm{SL}^{2} \text { was not entered because tolerance }\right. \\
\text { limits reached) }\end{array}$ \\
\hline \multicolumn{7}{|c|}{ Nonlinear models: } \\
\hline $\begin{array}{l}\text { Logarithmic } \\
\text { model }\end{array}$ & -2624.033 & 2165.687 & 0.428 & 927.076 & $* * *$ & $\hat{Y}=-2624.033+2165.687 \log$ SL. \\
\hline $\begin{array}{l}\text { Exponential } \\
\text { model }\end{array}$ & 1297.424 & 0.0018 & 0.005 & 0.906 & NS & \\
\hline \multicolumn{7}{|c|}{ All duck males } \\
\hline \multicolumn{7}{|c|}{ Polynomial models: } \\
\hline $\begin{array}{l}\text { Quadratic } \\
\text { model }\end{array}$ & -2286.386 & $\begin{array}{l}52.273 \\
-0.007 \\
\end{array}$ & 0.747 & 558.423 & $\begin{array}{l}* * * \\
* * * \\
\end{array}$ & $\hat{\mathrm{Y}}=-2286.386+52.273 \mathrm{SL}-0.007 \mathrm{SL}^{2}$ \\
\hline $\begin{array}{l}\text { Cubic } \\
\text { model }\end{array}$ & -2252.156 & \begin{tabular}{|c|}
51.235 \\
$-1.03 \mathrm{E}^{.06}$
\end{tabular} & 0.748 & 557.571 & $\begin{array}{l}* * * \\
* * *\end{array}$ & $\begin{array}{l}\hat{\mathrm{Y}}=-2252.156+51.235 \mathrm{SL}-1.03^{\mathrm{E} .06} \mathrm{SL}^{3} \\
\left(\mathrm{SL}{ }^{2} \text { was not entered because tolerance }\right. \\
\text { limits reached) }\end{array}$ \\
\hline \multicolumn{7}{|c|}{ Nonlinear models: } \\
\hline $\begin{array}{l}\text { Logarithmic } \\
\text { model }\end{array}$ & -8217.617 & 2296.956 & 0.467 & 809.628 & $\begin{array}{l}* * * \\
* * *\end{array}$ & $\hat{\mathrm{Y}}=-8217.617+2296.956 \log \mathrm{SL}$. \\
\hline $\begin{array}{l}\text { Exponential } \\
\text { model }\end{array}$ & 1246.007 & 0.0002 & 0.005 & 0.872 & $*$ & $\hat{\mathrm{Y}}=1246.007+(0.0002)^{S L}$ \\
\hline
\end{tabular}

Fayoum J. Agric. Res. \& Dev., Vol.19, No.1, January, 2005 
Table 4. The parameters, coefficient of determination $\left(R^{2}\right)$ of $L B W$ when the linear equation $\hat{\mathbf{Y}}=\mathbf{a}+\mathbf{b} \mathbf{X}$ was used for predicting the average $\mathbf{L B W}$ in grams $(\hat{\mathbf{Y}})$ from age in $(X)$ of different types.

\begin{tabular}{|l|c|c|c|c|c|c|c|}
\hline Group & Sex & $\mathbf{a}$ & $\mathbf{b}$ & $\mathbf{r}$ & $\mathbf{R}^{2}$ & Sig. & Fitted equation \\
\hline Fayoumi, PP & $\mathrm{M}$ & -12.773 & 9.218 & 0.946 & 0.895 & $* * *$ & $\hat{\mathrm{Y}}=-12.773+9.218 \mathrm{X}$ \\
\hline & $\mathrm{F}$ & 1.855 & 7.398 & 0.943 & 0.889 & $* * *$ & $\hat{\mathrm{Y}}=1.855+7.398 \mathrm{X}$ \\
\hline & $\mathrm{M}+\mathrm{F}$ & -6.084 & 8.327 & 0.935 & 0.873 & $* * *$ & $\hat{\mathrm{Y}}=-6.084+8.327 \mathrm{X}$ \\
\hline & $\mathrm{M}$ & -26.252 & 9.671 & 0.962 & 0.926 & $* * *$ & $\hat{\mathrm{Y}}=-26.252+9.671 \mathrm{X}$ \\
\hline & $\mathrm{F}$ & -8.974 & 7.694 & 0.960 & 0.921 & $* * *$ & $\hat{\mathrm{Y}}=-8.974+7.694 \mathrm{X}$ \\
\hline & $\mathrm{M}+\mathrm{F}$ & -17.043 & 8.617 & 0.948 & 0.899 & $* * *$ & $\hat{\mathrm{Y}}=-17.043+8.617 \mathrm{X}$ \\
\hline Golden Montazah & $\mathrm{M}$ & -52.334 & 15.405 & 0.982 & 0.964 & $* * *$ & $\hat{\mathrm{Y}}=-52.334+15.405 \mathrm{X}$ \\
\hline & $\mathrm{F}$ & -25.524 & 12.309 & 0.983 & 0.966 & $* * *$ & $\hat{\mathrm{Y}}=-25.524+12.309 \mathrm{X}$ \\
\hline s & $\mathrm{M}+\mathrm{F}$ & -39.397 & 13.911 & 0.970 & 0.940 & $* * *$ & $\hat{\mathrm{Y}}=-39.397+13.911 \mathrm{X}$ \\
\hline All chickens & $\mathrm{M}$ & -32.40 & 11.315 & 0.912 & 0.832 & $* * *$ & $\hat{\mathrm{Y}}=-32.400+13.315 \mathrm{X}$ \\
\hline & $\mathrm{F}$ & -12.487 & 8.904 & 0.899 & 0.809 & $* * *$ & $\hat{\mathrm{Y}}=-12.487+8.904 \mathrm{X}$ \\
\hline & $\mathrm{M}+\mathrm{F}$ & -22.444 & 10.091 & 0.912 & 0.832 & $* * *$ & $\hat{\mathrm{Y}}=-22.444+10.091 \mathrm{X}$ \\
\hline Pekin ducks & $\mathrm{M}$ & 5.764 & 34.555 & 0.932 & 0.868 & $* * *$ & $\hat{\mathrm{Y}}=5.764+34.555 \mathrm{X}$ \\
\hline & $\mathrm{F}$ & 73.452 & 31.274 & 0.916 & 0.839 & $* * *$ & $\hat{\mathrm{Y}}=73.452+31.274 \mathrm{X}$ \\
\hline & $\mathrm{M}+\mathrm{F}$ & 37.004 & 33.042 & 0.822 & 0.851 & $* * *$ & $\hat{\mathrm{Y}}=37.004+33.042 \mathrm{X}$ \\
\hline Moscuvy ducks & $\mathrm{M}$ & -713.071 & 59.499 & 0.964 & 0.930 & $* * *$ & $\hat{\mathrm{Y}}=-713.071+59.499 \mathrm{X}$ \\
\hline & $\mathrm{F}$ & -346.305 & 38.648 & 0.965 & 0.932 & $* * *$ & $\hat{\mathrm{Y}}=-346.305+38.648 \mathrm{X}$ \\
\hline & $\mathrm{M}+\mathrm{F}$ & -585.944 & 50.369 & 0.917 & 0.841 & $* * *$ & $\hat{\mathrm{Y}}=-585.944+50.369 \mathrm{X}$ \\
\hline All ducks & $\mathrm{M}$ & -416.135 & 49.031 & 0.836 & 0.914 & $* * *$ & $\hat{\mathrm{Y}}=-416.135+49.031 \mathrm{X}$ \\
\hline & $\mathrm{F}$ & -208.039 & 36.070 & 0.892 & 0.944 & $* * *$ & $\hat{\mathrm{Y}}=-208.039+36.070 \mathrm{X}$ \\
\hline & $\mathrm{M}+\mathrm{F}$ & -339.515 & 43.307 & 0.808 & 0.899 & $* * *$ & $\hat{\mathrm{Y}}=-339.515+43.307 \mathrm{X}$ \\
\hline Japanese Quail & $\mathrm{M}$ & -7.999 & 4.320 & 0.981 & 0.962 & $* * *$ & $\hat{\mathrm{Y}}=-7.959+4.320 \mathrm{X}$ \\
\hline & $\mathrm{F}$ & -9.400 & 4.628 & 0.976 & 0.953 & $* * *$ & $\hat{\mathrm{Y}}=9.400+4.628 \mathrm{X}$ \\
\hline & $\mathrm{M}+\mathrm{F}$ & -8.743 & 4.483 & 0.977 & 0.954 & $* * *$ & $\hat{\mathrm{Y}}=-8.743+4.483 \mathrm{X}$ \\
\hline & & & & & &
\end{tabular}

Sig : significance and ${ }^{* * *}$ : significantly different at $\mathrm{P} \leq 0.001, \mathrm{M}, \mathrm{F}$ and $\mathrm{M}+\mathrm{F}$ :see footnote in Table3.

Similarly, trends of positive significant influence of age in days on SL in millimeters but lower in magnitude than LBW were observed in most studied groups or sex groups, except for SL of Moscuvy males, combined sexes of Moscuvy ducks and all duck females which were insignificantly affected by age. The rate of increase in SL which ranged from $0.418 \mathrm{~mm}$ in case of Japanese quail females to $0.936 \mathrm{~mm}$ in GM males as shown in Table 5.

Comparing the coefficient of determination in the three studied linear models, it can be seen that higher ' $\mathrm{R}^{2}$ ' values were observed for the second (predicting LBW through age) and first (predicting LBW through SL) models than the third (predicting SL through age) model.

In conclusion, there were definite relationships between LBW and SL and either LBW or SL significantly were affected by bird age. Comparing ' $R$ ', of fitted equations indicated that linear model was found to be suitable for predicting LBW through bird age or SL. Although all traits could be used to predict live body weight, the use of age $(\mathrm{P} \leq 0.001)$ yielded an $\mathrm{R}^{2}$ which ranged from 0.809 to 0.966 . In the absence of age, the use of shank length yielded an $R^{2}$ which ranged

Fayoum J. Agric. Res. \& Dev., Vol.19, No.1, January, 2005 
PREDICTION OF LIVE BODY WEIGHT THROUGH BIRD AGE

from 0.630 to 0.955 . It can be concluded that in the absence of age, shank length can be used to predict live body weight.

Table 5. The parameters, coefficient of determination $\left(R^{2}\right)$ of $L B W$ when the linear equation $\hat{\mathbf{Y}}=\mathbf{a}+\mathbf{b} \mathbf{X}$ was used for predicting the average SL $(\hat{\mathbf{Y}})$ from age in (X) of different types.

\begin{tabular}{|c|c|c|c|c|c|c|c|}
\hline Group & Sex & $\mathbf{a}$ & $\mathbf{b}$ & $\mathbf{r}$ & $\mathbf{R}^{2}$ & Sig. & Fitted equation \\
\hline Fayoumi, PP & $\mathrm{M}$ & 33.738 & 0.724 & 0.940 & 0.883 & $* * *$ & $\hat{\mathrm{Y}}=33.738+0.724 \mathrm{X}$ \\
\hline & $\mathrm{F}$ & 34.109 & 0.611 & 0.928 & 0.860 & $* * *$ & $\hat{\mathrm{Y}}=34.109+0.611 \mathrm{X}$ \\
\hline & $\mathrm{M}+\mathrm{F}$ & 33.732 & 0.671 & 0.918 & 0.843 & $* * *$ & $\hat{\mathrm{Y}}=33.732+0.671 \mathrm{X}$ \\
\hline Dandarawi & $\mathrm{M}$ & 30.022 & 0.806 & 0.778 & 0.606 & $* * *$ & $\hat{\mathrm{Y}}=30.022+0.806 \mathrm{X}$ \\
\hline & $\mathrm{F}$ & 30.658 & 0.677 & 0.941 & 0.885 & $* * *$ & $\hat{\mathrm{Y}}=30.658+0.677 \mathrm{X}$ \\
\hline & $\mathrm{M}+\mathrm{F}$ & 30.361 & 0.737 & 0.826 & 0.682 & $* * *$ & $\hat{\mathrm{Y}}=33.361+0.737 \mathrm{X}$ \\
\hline Golden Montazah & $\mathrm{M}$ & 33.904 & 0.936 & 0.977 & 0.954 & $* * *$ & $\hat{\mathrm{Y}}=33.904+0.936 \mathrm{X}$ \\
\hline & $\mathrm{F}$ & 35.034 & 0.794 & 0.967 & 0.936 & $* * *$ & $\hat{\mathrm{Y}}=35.034+0.794 \mathrm{X}$ \\
\hline & $\mathrm{M}+\mathrm{F}$ & 34.449 & 0.868 & 0.956 & 0.915 & $* * *$ & $\hat{\mathrm{Y}}=34.449+0.868 \mathrm{X}$ \\
\hline & $\mathrm{M}$ & 31.833 & 0.829 & 0.846 & 0.716 & $* * *$ & $\hat{\mathrm{Y}}=31.833+0.829 \mathrm{X}$ \\
\hline All chickens & $\mathrm{F}$ & 32.365 & 0.698 & 0.908 & 0.824 & $* * *$ & $\hat{\mathrm{Y}}=32.365+0.698 \mathrm{X}$ \\
\hline & $\mathrm{M}+\mathrm{F}$ & 32.052 & 0.762 & 0.856 & 0.733 & $* * *$ & $\hat{\mathrm{Y}}=32.052+0.762 \mathrm{X}$ \\
\hline & $\mathrm{M}$ & 49.867 & 0.519 & 0.771 & 0.594 & $* * *$ & $\hat{\mathrm{Y}}=49.867+0.519 \mathrm{X}$ \\
\hline & $\mathrm{F}$ & 51.346 & 0.435 & 0.752 & 0.566 & $* * *$ & $\hat{\mathrm{Y}}=51.346+0.435 \mathrm{X}$ \\
\hline Pekin ducks & $\mathrm{M}+\mathrm{F}$ & 50.542 & 0.480 & 0.759 & 0.576 & $* * *$ & $\hat{\mathrm{Y}}=50.542+0.480 \mathrm{X}$ \\
\hline & $\mathrm{M}$ & 61.598 & 0.936 & 0.048 & 0.002 & $\mathrm{NS}$ & \\
\hline Moscuvy ducks & $\mathrm{F}$ & 37.472 & 0.819 & 0.887 & 0.787 & $* * *$ & $\hat{\mathrm{Y}}=37.472+0.819 \mathrm{X}$ \\
\hline & $\mathrm{M}+\mathrm{F}$ & 46.470 & 0.932 & 0.066 & 0.004 & $\mathrm{NS}$ & \\
\hline & $\mathrm{M}$ & 41.756 & 0.702 & 0.841 & 0.708 & $* * *$ & $\hat{\mathrm{Y}}=41.756+0.702 \mathrm{X}$ \\
\hline All ducks & $\mathrm{F}$ & 55.666 & 0.840 & 0.051 & 0.003 & $\mathrm{NS}$ & \\
\hline & $\mathrm{M}+\mathrm{F}$ & 47.361 & 0.802 & 0.068 & 0.005 & $*$ & $\hat{\mathrm{Y}}=47.361+0.802 \mathrm{X}$ \\
\hline & $\mathrm{M}$ & 22.689 & 0.462 & 0.879 & 0.772 & $* * *$ & $\hat{\mathrm{Y}}=22.689+0.462 \mathrm{X}$ \\
\hline Japanese Quail & $\mathrm{F}$ & 24.235 & 0.418 & 0.894 & 0.800 & $* * *$ & $\hat{\mathrm{Y}}=24.235+0.418 \mathrm{X}$ \\
\hline & $\mathrm{M}+\mathrm{F}$ & 23.510 & 0.439 & 0.885 & 0.783 & $* * *$ & $\hat{\mathrm{Y}}=23.510+0.439 \mathrm{X}$ \\
\hline
\end{tabular}

Sig : significance and $* * *$ : significantly different at $\mathrm{P} \leq 0.001, \mathrm{M}, \mathrm{F}$ and $\mathrm{M}+\mathrm{F}$ :see footnote in Table3.

\section{REFERENCES}

Chambers, J. R. (1993). Genetics of growth and meat production in chickens. pp: 599-643. Poultry Breeding and Genetics, edited by R.D. Crawford, Elsevier Science, Amsterdam, The Netherlands.

Egyptian Ministerial Decree No. 1498 (1996). Ministerial decree No. 1498 of the year 1996 concerning Foodstuffs industry in Egypt.

EI Full, E. and Farahat, G. S. (2004). Prediction of body weight at eight weeks of age through body measurements at different ages for Muscovy ducklings. Fayoum J. Agric. Res. \& Dev., 18: 67-74.

Jaap, R. G. and Penquite, R. (1938). Criteria of conformation in market poultry. Poult. Sci. 17:425-430.

Jaap, R. G. and Thomson, R. B. (1940). Heritable differences in conformation of adult female fowl. Poult. Sci.19:73-78.

Kanoun, A.H. (1984). A. B. A, 52 (1-3):1197. Cited from : Sunanda, C., George, L. C. and Nair, G. R. (1991). Prediction of LBW through SL in Desi ducks. Cherion $20: 9-12$.

Fayoum J. Agric. Res. \& Dev., Vol.19, No.1, January, 2005 
Knizetova, H., Hyanek, J. and Veselsky (1994). Analysis of growth curves of fowl: III. Geese. Br. Poult. Sci. 35:335-344.

Kumar, P. Verma, S. B., Mandal, K. G., Akhtar, S.M.H. and Kumar, P. (2002). Influence of sex on body weight and conformation traits in a random bred population of Japanese quail (Coturnix coturnix Japonica). Indian J. Anim. Health. 41: 61-63.

N R C (1994). Nutrient requirements of poultry. National Research Councel, $9^{\text {th }}$ revised ed. Nat. Acad. Press, Washington, D. C.

Petek, M. Balci, F. Baspinar, H. Ogan, M. and Dikmen, S. (2000). The effects of shank length on some production traits of a commercial layer flock. Veteriner Fakultesi Dergisi Uludag Universitesi. 19:129-134.

Shanawany, M.M. and Morris, T.R. (1992). The regression of body weight on shank length in chickens. World Review of Animal Production. 27:91-93.

Singh, A., Chaudhary, M.L., Brah, G.S. and Sandhu, J. S. (2001). Inheritance of external body measurements and their relationships with economic traits in layer chickens. Indian J. Poult. Sci. 36: 153-157.

Steel, R. G. and Torrie, J. H. (1981). Principals and Procedures of Statistics. $2^{\text {nd }}$ Ed. McGraw Hill Book Co., New York, USA.

Sunanda, C. , George, L. C. and Nair, G. R. (1991). Prediction of LBW through SL in Desi ducks. Cherion 20: 9-12.

Tierce, J. and Nordskog, A.W. (1985). Poult. Sci. 64 : 605. Cited from: Sunanda, C., George, L. C. and Nair, G. R. (1991). Prediction of LBW through SL in Desi ducks. Cherion 20: 9-12.

Verma, S.K., Sharma, S.D. and Mishra, H.B. (1977). Indian Poult. Gazzette 61(2) :68. Cited from : Sunanda, C., George, L. C. and Nair, G. R. (1991). Prediction of LBW through SL in Desi ducks. Cherion 20: 9-12.

Wise, D.R. (1970). Carcass conformation comparisons of growing broiler and laying strain chickens. Br. Poult. Sci 11:325-332.

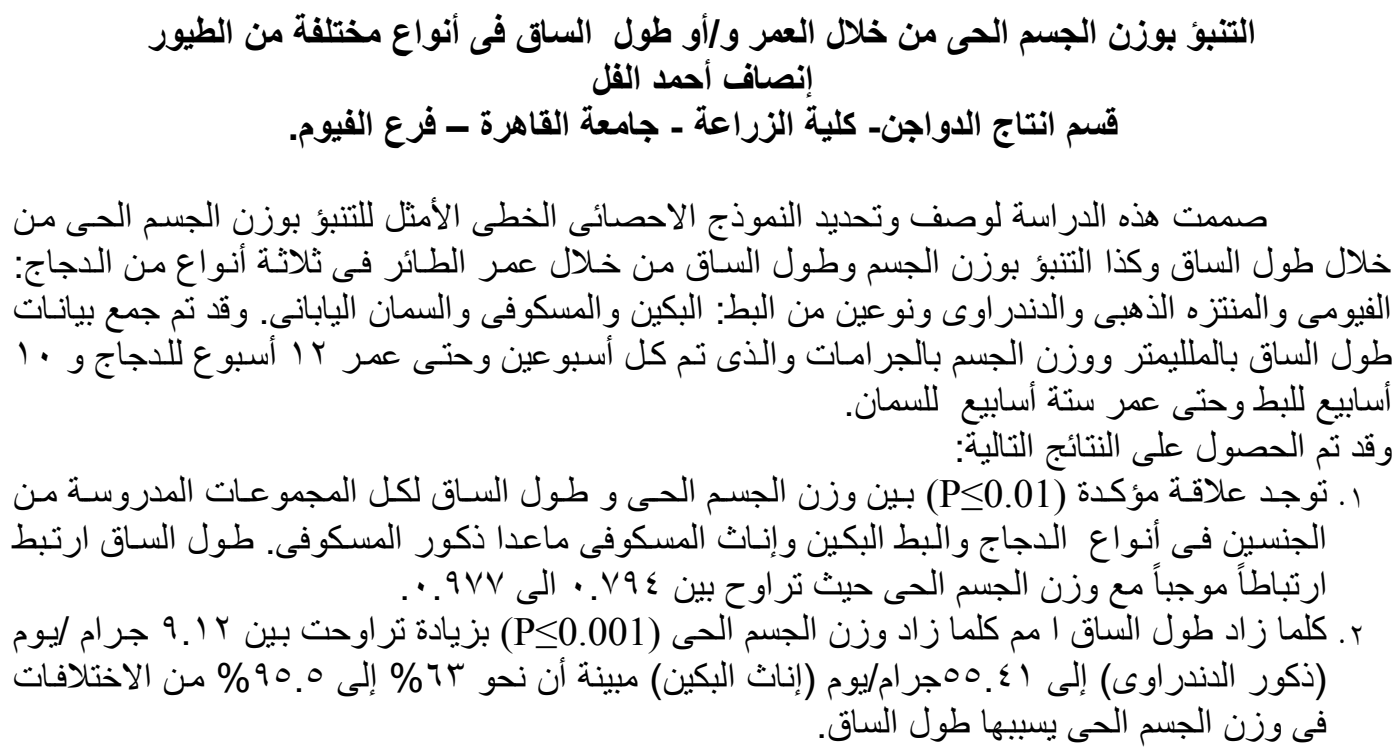

Fayoum J. Agric. Res. \& Dev., Vol.19, No.1, January, 2005 


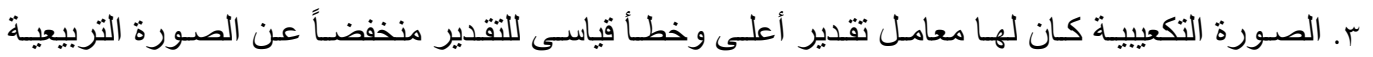

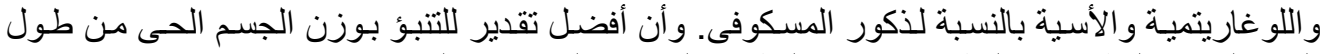

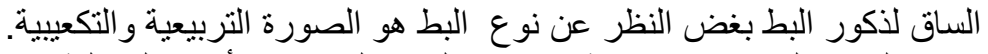

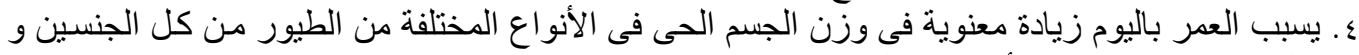

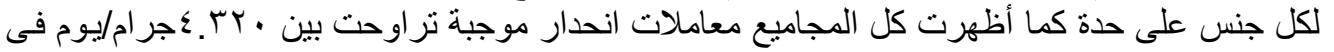

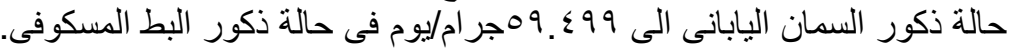

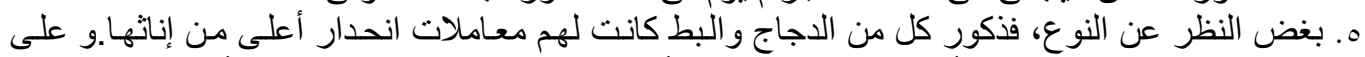

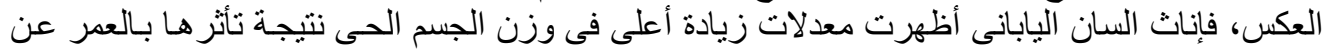
ذكور ها.

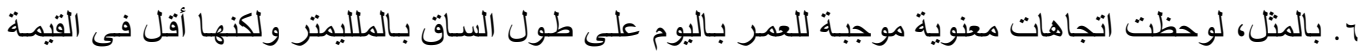

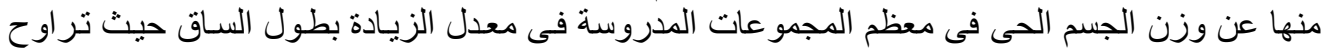

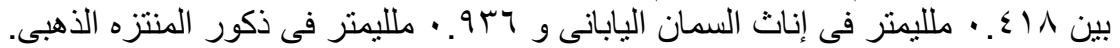

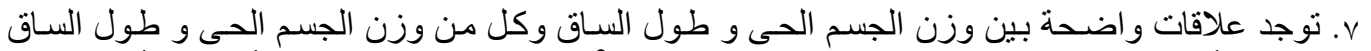

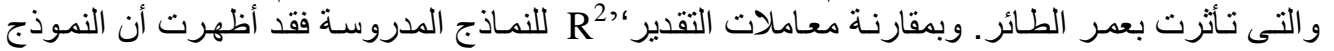

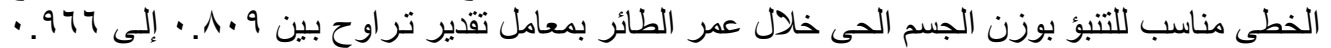

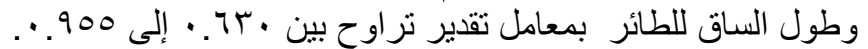

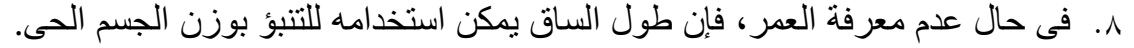

